\title{
Control of performance by short-term memory in honeybees
}

\author{
P. A. COUVILLON, N. M. ARINCORAYAN, and M. E. BITTERMAN \\ University of Hawaii, Honolulu, Hawaii
}

\begin{abstract}
Individual honeybees foraging at a laboratory window were trained with a correction method to choose between blue and yellow targets, one of which contained sucrose solution. There were two trials on each visit, with the locus of the sucrose predictable only on the second. Animals differentially rewarded on Trial 2 for choosing the rewarded color of Trial 1 , for choosing the alternative color, or for choosing the target in the rewarded position of Trial 1 independently of its color, all showed a small but persistent preference for the rewarded color, with no significant preference for the rewarded position. When the positions of the colored targets were the same on Trial 2 as on Trial 1 (color and position confounded), there was a more substantial but equally persistent preference on Trial 2 for the rewarded color-position of Trial 1, whether the animals were differentially rewarded for perseveration or for alternation. The results provide further evidence of unlearned control of performance by short-term memory in honeybees but no indication of learned control.
\end{abstract}

In a search for evidence of the control of performance by short-term memory in honeybees, Ohyama, Couvillon, and Bitterman (1995) trained individual foragers with targets of two different colors, rewarding them on each trial for choosing the nonrewarded color of the immediately preceding trial. The training situation, diagramed in Figure 1, was a resined plywood enclosure set into a laboratory window. On each trial, the animal chose between blue and yellow targets in positions $\mathrm{B}$ and $\mathrm{C}$, sometimes in one of the two possible spatial arrangements and sometimes in the other. One of the targets, sometimes the blue and sometimes the yellow, contained a 10- $\mu$ l drop of sucrose solution. When the animal found the sucrose, say, in the blue target, that target with the animal feeding in it was moved to A. Then, after ingesting the sucrose, the animal chose between fresh blue and yellow targets that, in the meantime, had been introduced at B and C, in either the same or the opposite spatial arrangement as before, with the yellow target now containing sucrose; and so forth. The training continued in this way until the animal was replete, whereupon it left of its own accord for the hive to empty its social stomach, returning several minutes later for another set of trials (usually four or five per visit).

Although color alternation was differentially rewarded, the honeybees did not learn to alternate. Instead, they showed from the outset a small but reliable tendency to perseverate in the choice of color-that is, to choose on

This work was supported, in part, by Grant IBN-9601991 from the National Science Foundation and, in part, by Research Centers for Minority Institutions Grant RR03061 from the National Institutes of Health. The participation of N.M.A. was made possible by Minority Access to Research Careers Grant GM07584-18 from the National Institutes of Health. Correspondence should be addressed to M. E. Bitterman, Békésy Laboratory of Neurobiology, 1993 East-West Road, Honolulu, HI 96822 (e-mail: jeffb@pbrc.hawaii.edu). each trial (with a probability of about .6) the rewarded color of the immediately preceding trial. There was perseveration also in the choice of position. Although differentially rewarded neither for spatial alternation nor for spatial perseveration, the honeybees showed from the outset a small but reliable tendency to go on each trial (with a probability of about .6) to the rewarded position of the immediately preceding trial. In a subsequent experiment with targets of a single color, Isnec, Couvillon, and Bitterman (1997) found the same small but reliable tendency to perseverate in the choice of position, whether the animals were differentially rewarded for alternation or rewarded for either choice.

If honeybees could be trained to alternate (whether in the choice of color or of position), control of performance by short-term memory would be indicated; as Dennis (1939) noted in an early paper on spontaneous spatial alternation in rats, in order to avoid the more recently encountered of two alternatives-for whatever reason-an animal must, in some sense, remember which it was. For perseveration, however, a simpler explanation is conceivable, the attractiveness of each alternative being assumed to depend on the strength of an association with sucrose that increases on each rewarded trial and decreases on each nonrewarded trial, just as in conventional discriminative training (Couvillon \& Bitterman, 1991). Isnec et al. (1997) rejected an associative interpretation of the spatial perseveration shown by their animals, on the basis of the results of companion experiments in which the same position was rewarded over a series of consecutive trials. Stable preferences that could be interpreted as associative developed as expected but much too slowly to explain the trialto-trial perseveration observed in alternation training; the strength of the initial tendency to return to a rewarded position increased substantially in the course of eight consecutive rewards at that position but not at all after only 


\section{EXPERIMENTER \\ (INSIDE LABORATORY)}

SLIDING PLEXIGLAS DOORS

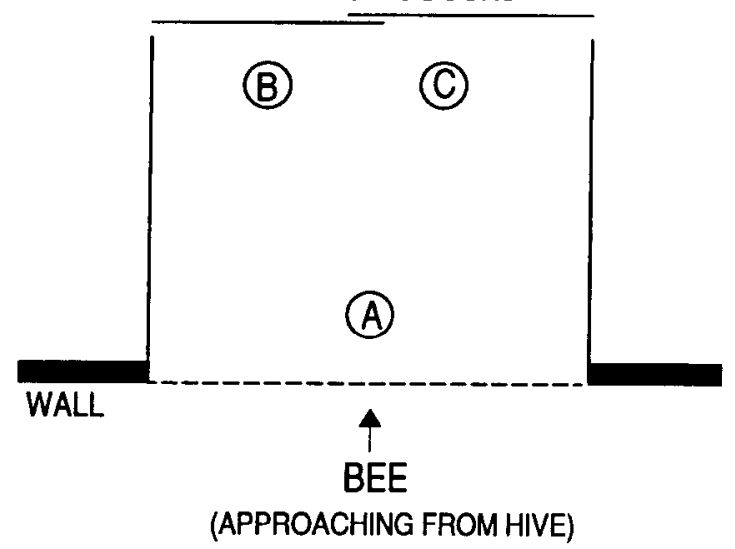

Figure 1. Diagram of the experimental situation showing the locations $(A, B$, and $C)$ at which targets were placed during training. The enclosure ( $58 \mathrm{~cm}$ wide, $58 \mathrm{~cm}$ high, and $46 \mathrm{~cm}$ deep) was open to the outside, and it was fitted with a pair of sliding Plexiglas panels, through which the observer had access from the inside. The center-to-center distance from $A$ to $B$ and from $A$ to $C$ was $27 \mathrm{~cm}$; the distance from $B$ to $C$ was $16 \mathrm{~cm}$.

four. To account for all the results, it seemed necessary to assume a short-term memory process as well as an associative process, the first discontinuous and transient, the second gradual and relatively stable, and both tending to produce perseveration.

An interesting and possibly divergent aspect of the honeybee results is that the perseverative tendency attributable to short-term memory, seemed not only unlearned (evident at the very outset of training) but unmodifiable (entirely undiminished by differential reward for alternation). In the present experiment, we looked further for evidence of learned control of performance by short-term memory, with a procedure designed to minimize proactive interference. Instead of multiple trials on each visit to the laboratory, as in the previous experiments, there were now only two trials on each visit, the second providing enough sucrose solution to fill the social stomach and to prompt return to the hive. The locus of reward on the first trial, which varied in quasi-random fashion from visit to visit, served to inform the animal as to the locus of reward on the second trial. The second trial constituted a test of the subject's ability both to retain that information in the short interval between trials and to learn to use it appropriately. It may be important that the two-trial procedure involved an increase in the amount of reward for correct response, which, in previous experiments (with multiple trials per visit), was of necessity small; here, the reward on Trial 2 was feeding to repletion, and the possibility was considered that learned control of performance by short-term memory might, in consequence, be facilitated.
A second noteworthy feature of the present experiment is that not only were some animals rewarded for alternation, as in our previous experiments, but other animals were rewarded for perseveration. Just as the alternation training might be expected to reduce or even to reverse any initial tendency to perseverate, so the perseveration training might be expected to strengthen it. A color perseveration group (Group CP), which was rewarded on Trial 2 for choosing the rewarded color of Trial 1 independently of its position, was compared with a color alternation group (Group CA), which was rewarded on Trial 2 for choosing the alternative color independently of its position. There was also a position perseveration group (Group PP), which was rewarded on Trial 2 for choosing the target in the rewarded position of Trial 1 independently of its color. In the training of these groups, as in our previous experiment on color alternation (Ohyama et al., 1995), the positions of the two colored targets (at B and $\mathrm{C}$ in Figure 1) were interchanged quasi-randomly, both from trial to trial within visits and from visit to visit.

A third noteworthy feature of the present experiment is that other animals were trained with the positions of the colored targets always the same on Trials 1 and 2 of any given visit; that is, color and position were confounded within visits. A confounded perseveration group (Group CfP) was rewarded on Trial 2 for choosing the rewarded color-position of Trial 1, and two confounded alternation groups (Groups CfA and CfAi) were rewarded for choosing the alternative color-position. For the animals of Groups CP, CA, and PP, tendencies to choose in terms of color or position would be compatible whenever the positions of the colors happened to be the same on Trial 2 as they were on Trial 1 of a given visit, but incompatible when the positions had been interchanged. For the three confounded groups, however, the tendencies would always be compatible, the only options available on Trial 2 of any visit being to choose either the rewarded color-position of Trial 1 or the alternative color-position. The possibility was considered that learned control of performance by short-term memory might be established more readily in the confounded training.

A fourth noteworthy feature of the present experiment is to be found in the training of Group CfAi. For Groups CP, $\mathrm{CA}$, and $\mathrm{PP}$, the positions of the colors were interchanged quasi-randomly both within and between visits; for Groups CfP and CfA, the positions of the colors were interchanged quasi-randomly only between (not within) visits; but for Group CfAi, the positions of the colors were invariant both within and between visits - that is, each CfAi subject encountered the colors only in one of their two possible spatial arrangements. The CfAi procedure was designed to permit a systematic comparison of perseveration in the short intervals between trials within visits (from Trial 1 to Trial 2 of each visit) and perseveration in the longer intervals between visits (from Trial 2 of one visit to Trial 1 of the next). The explanation of perseveration in terms of short-term memory suggested that perseveration should be less in the longer interval. 


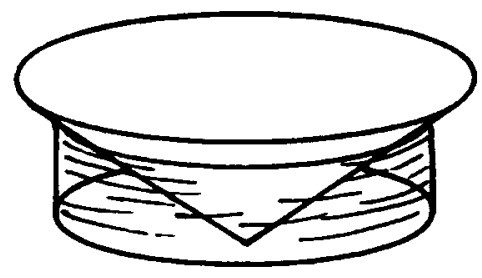

Figure 2. Sketch of a target $(6.2 \mathrm{~cm}$ in its widest diameter, $2 \mathrm{~cm}$ deep, and $28 \mathrm{ml}$ in volume). Each target was a plastic coneyellow or blue - that was set in a clear cylindrical base.

\section{METHOD}

\section{Subjects}

The subjects were 48 foraging honeybees (Apis mellifera), all of them experimentally naive, from our own hives situated near the laboratory. They were assigned quasi-randomly to six groups of 8 animals each. The animals were trained individually and sequentially, each in a single session of several hours duration.

\section{Procedure}

The training situation was the same as that diagramed in Figure 1-. a resined plywood enclosure, $58 \mathrm{~cm}$ wide, $58 \mathrm{~cm}$ high, and $46 \mathrm{~cm}$ deep, set into a laboratory window. The enclosure was open to the outside, and it was fitted with a pair of sliding Plexiglas panels, through which the observer had access to the enclosure from the inside. The targets used were plastic cones like that diagramed in Figure $2,6.2 \mathrm{~cm}$ in the widest diameter, $2 \mathrm{~cm}$ high, and $28 \mathrm{ml}$ in volume. Their colors were either blue or yellow. The center-to-center distance between targets in positions labeled $\mathrm{A}$ and $\mathrm{B}$ in Figure 1 was $27 \mathrm{~cm}$, and so also was the distance between $\mathrm{A}$ and $\mathrm{C}$; the distance from $\mathrm{B}$ to $\mathrm{C}$ was $16 \mathrm{~cm}$.

Each subject was captured in a match box on its arrival at a feeding station providing $10 \%-15 \%$ sucrose solution, carried to the laboratory, and set down at a large $(\sim 100-\mu 1)$ drop of $50 \%$ sucrose solution in a single target (blue for half the animals in each group and yellow for the rest) at position $\mathrm{A}$ in the diagram. The subject was marked with a spot of colored lacquer as it fed to repletion, after which it would leave for the hive to deposit the sucrose. Normally, the animal would come back to the laboratory several minutes later, continuing thereafter to shuttle between the hive and the training situation as long as sucrose was provided there. If it did not come back after its first placement, it was carried again to the training situation from the feeding station, where it usually could be found. When the animal did come back of its own accord, it found another baited target at A (yellow if the first was blue and blue if the first was yellow), from which it fed to repletion. The targets used on these pretraining visits and on the subsequent training visits were drawn from two large sets of otherwise identical targets, to which they were returned after washing with water at the conclusion of the visit; the purpose of the procedure (a standard one in this laboratory) is to randomize extraneous stimuli. The use of fresh targets for each choice is especially critical in an experiment such as this one, because of the tendency of foragers to mark depleted flowers with an aversive scent (Giurfa \& Núñez, 1992; Núñez, 1967).

Arriving from the hive on each of 24 training visits, the animal found blue and yellow targets in positions $\mathrm{B}$ and $\mathrm{C}$. For all groups except Group CfAi, the colors were in one spatial arrangement (e.g., by, designating blue at $\mathrm{B}$ and yellow at $\mathrm{C}$ ) on half the visits and in the opposite spatial arrangement $(y b$, designating yellow at $\mathrm{B}$ and blue at $\mathrm{C}$ ) on the rest, in balanced quasi-random order. For Group $\mathrm{CfAi}$, the arrangement always was the same- $b y$ for half the animals and $y b$ for the rest. On half the visits for all groups, the blue target contained a $10-\mu$ drop of $50 \%$ sucrose solution, and the yel- low target contained a 10- $\mu \mathrm{l}$ drop of water; on the remaining visits, sucrose was in the yellow, and water was in the blue. The role of the water, which was unacceptable and which could be distinguished from the sucrose only by taste, was to eliminate the possibility that the correct target was discriminable on the basis of the presence of the drop of sucrose it contained. To reach the sucrose or the water, the animal was obliged to land on the inside surface of the target and walk down to the bottom, and, if the animal went first to the water, it was free at once to correct its choice. The experimenter recorded whether the initial response of the animal was correct or incorrect, incorrect choice being defined as a full landing on the inside surface of the target containing water.

After the animal found the sucrose in one of the targets, that target with the animal feeding in it was moved to position $\mathrm{A}$, and fresh blue and yellow targets were introduced at $B$ and $C$ for a second choice trial. For Groups CP, CA, and PP, the spatial arrangement remained the same on half the visits ( $b y$ if $b y$ and $y b$ if $y b$ ) but was reversed on the rest ( $y b$ if $b y$ and $b y$ if $y b$ ), in quasi-random order. For the confounded groups (Groups CfP, CfA, and CfAi), the spatial arrangement of the colors was always the same on the second trial as it was on the first. The geometry of the situation (the structure of the targets together with the orientation of the animal) was such that the animal, as it continued to feed, could not see either the targets being introduced or the carefully limited movements of the experimenter. After ingesting the sucrose, the animal would fly up and then choose between the fresh targets, one of which contained a large $(\sim 100-\mu 1)$ drop of $50 \%$ sucrose solution and the other a large drop of water. For Group CP, the sucrose was to be found in the target of the same color as on arrival, irrespective of position; for Group CA, in the target of the other color; and for Group PP, in the same position as on arrival, irrespective of the color of the target in that position. For Group CfP, the sucrose was to be found in the same color-position as on arrival; and for Groups CfA and CfAi, in the alternative color-position. Again, correction was permitted, and, after finding the sucrose solution and feeding to repletion, the animal would leave for the hive, coming back to the laboratory window several minutes later for another pair of trials.

\section{RESULTS}

As expected, the probability of correct choice on Trial 1 of each visit began and remained at the chance level for all six groups; the overall probabilities of correct initial choice ranged from .43 to .55 , with $t$-values ( $d f=7$, twotailed) ranging from 0.28 to 2.16 , in no case differing significantly $(p>.05)$ from the chance probability of .5 (the $\alpha$ level employed throughout was .05). The training procedure was, of course, so designed that there could be no basis for predicting the locus of reward on Trial 1.

On Trial 2, Groups CP, CA, and PP were alike in showing a modest tendency to perseverate in the choice of color; that is, they tended on Trial 2 to prefer the color rewarded on Trial 1. It did not matter whether they were differentially rewarded for perseveration in the choice of color (Group CP), for color alternation (Group CA), or for perseveration in the choice of position independently of color (Group PP). The probabilities of perseveration in the choice of color, ranging from .62 to .64 and each significantly greater than .5 ( $t$-values ranging from 2.68 to 5.83 ), are plotted in Figure 3. The three groups were alike also in showing very little tendency to perseverate in the choice of position, whether such perseveration was differentially rewarded (Group PP) or nondifferentially re- 


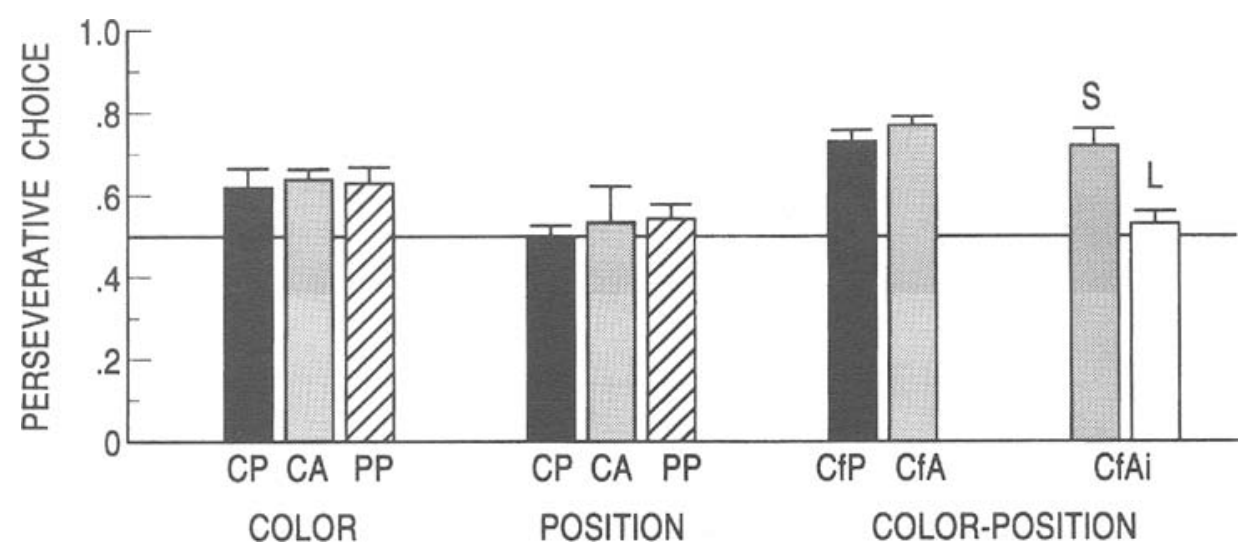

Figure 3. Mean proportions of perseverative choices (with standard errors) for the six groups. For Group CfAi, perseveration after the short intra-visit intervals $(S)$ is compared with perseveration after the longer inter-visit intervals $(L)$.

warded (Groups CP and CA). The probabilities of perseveration in the choice of position, ranging from .50 to .54 and none of them significantly different from .5 ( $t$-values ranging from 0.04 to 1.28), are plotted in Figure 3. A group $\times$ dimension $\times$ block analysis of variance (ANOVA) yields a significant dimension (color vs. position) effect $[F(1,21)=5.37]$, with no significant group effect $(F<1)$, no significant group $\times$ dimension interaction $(F<1)$, no significant change over six successive four-visit blocks $[F(5,105)=1.31]$, no significant group $\times$ block interaction $[F(10,105)=1.32]$, and no significant dimension $\times$ block interaction $[F(10,105)=1.30]$.

The confounded groups showed a marked tendency to perseverate in the choice of color-position on Trial 2, whether differentially rewarded for perseveration (Group CfP) or for alternation (Groups CfA and CfAi). The three probabilities are plotted in Figure 3 (the CfP bar; the CfA bar; and the CfAi bar labeled S, which shows the within-visits probability of perseveration in that group). A $2 \times 2$ ANOVA based on the data of the two color groups (Groups CP and CA) and the two confounded groups for which the positions of the colors also varied from visit to visit (Groups CfP and CfA) points to significantly greater perseveration in the confounded groups $(F=13.18)$, with no significant effect of training contingency (reward for perseveration vs. reward for alternation; $F<1$ ) and no significant interaction of confounding $\times$ training contingency $(F<1)$.

In Figure 4, the performance of Groups CfP and CfA (for both of which the spatial arrangement of the colors was varied between visits) is plotted in terms of the proportion of animals in each group choosing correctly on the second trial of each of the 24 training visits. As the curves indicate, a persistent tendency on the part of the animals to perseverate in the choice of color-position produced probabilities of correct choice that continued to be substantially better than chance for the perseveration group (Group CfP) and poorer than chance to the same extent for the alternation group (Group CfA). An ANOVA yields a significant group effect $[F(1,14)=176.72]$, with no significant change in performance over six successive four-visit blocks $[F(5,70)=1.17]$, and no significant group $\times$ block interaction $(F<1)$.

As is shown in Figure 3, perseveration in the choice of color-position on Trial 2 of each visit (perseveration from Trial 1 to Trial 2) was the same in Group CfAi (the bar labeled S), for which the color arrangement was the same on all visits, as it was in the other two confounded groups, for which the color-position arrangement varied quasi-randomly from visit to visit. As Figure 3 also shows, however, there was no tendency on the part of the CfAi animals (the bar labeled L) to perseverate over the much longer intervals between visits (from Trial 2 of one visit to Trial 1 of the next), which averaged $207 \mathrm{sec}(S E=$ 19.4), as compared with within-visit intervals (unrecorded) on the order of 5-10 sec. The probability of perseveration in the choice of color-position on Trial 1 (.53) did not differ significantly from chance $[t(7)=$ $0.87]$.

\section{DISCUSSION}

The animals of Group CA showed, from the outset, a small but reliable tendency to perseverate in the choice of color that did not decrease in the course of continued alternation training. Their performance was like that reported previously by Ohyama et al. (1995) for a group of animals trained under identical conditions, except with more trials per visit and an amount of reward for each correct choice that was of necessity considerably smaller. The performance of Groups CP and PP, the former differentially rewarded for perseverating in the choice of color independently of position and the latter for perseverating in the choice of position independently of color, 


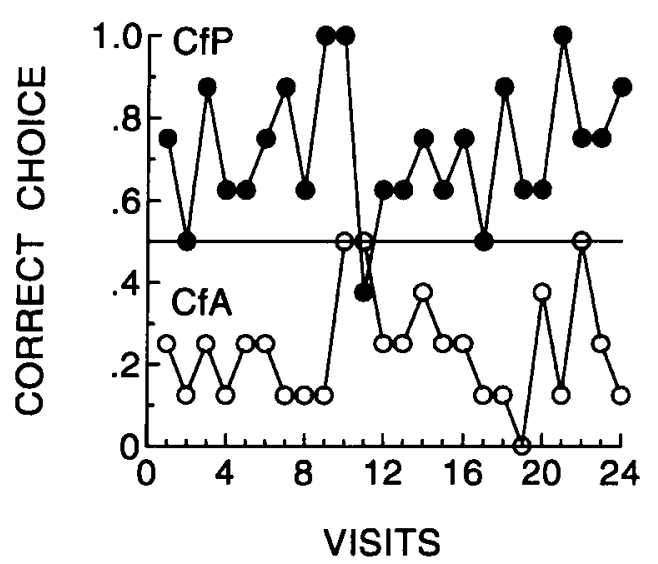

Figure 4. The proportion of animals in Groups $\mathrm{CfP}$ and $\mathrm{CfA}$ choosing correctly on the second trial of each visit.

proved to be indistinguishable from that of Group CA, confirming again both the existence of a small original tendency to perseverate in the choice of color and its insensitivity to contingencies of reinforcement.

A difference between the performance of Group CA and the performance of the color alternation group previously trained by Ohyama et al. (1995) is that the CA animals showed no significant tendency to perseverate in choice of position. In this respect, too, Groups CA, CP, and $\mathrm{PP}$ were indistinguishable, despite the fact that the PP animals were differentially rewarded for perseveration in the choice of position. It is clear, both from the results of the previous experiment and from the performance of the confounded groups (whose perseveration in the choice of color-position was substantially greater than the perseveration in the choice of color shown by Groups $\mathrm{CP}$ and $\mathrm{CA}$ ), that position is readily discriminable in the situation employed, although it is not clear why the animals of Groups CP, CA, and PP should have shown no significant tendency to respond in terms of position. The answer may lie in the weakness of the tendency, together with the lower reliability attendant upon the relatively small number of measurements for each subject afforded by the present procedure - only one critical choice on each visit, as compared with four or five on each visit in the Ohyama et al. experiment.

The interpretation of the color effect is complicated, because the training procedure - adapted from Waddington and Gottlieb (1990) — was such that each choice was made in the presence of the most recently rewarded color. The possibility must, therefore, be considered that the perseveration in the choice of color was influenced, at least to some extent, by the continued presence of the color, as distinct from its short-term memory. To estimate the extent of that influence, it will be necessary to develop a technique that removes the color without, at the same time, introducing other difficulties, which we have not thus far done. As to the position effect, however, there is no such problem; with the animal at the choice point equidistant from the two alternatives, the basis of perseveration can only be mnemonic. Although the perseveration in the choice of position found in our previous experiments, both with uncorrelated variation in color (Ohyama et al., 1995) and with the two alternatives the same in color (Isnec et al., 1997), was not shown under the conditions of the present experiment by Groups CP, CA, and PP, the greater perseveration in the choice of color-position by the confounded groups, as compared with perseveration in the choice of color by Groups CP, CA, and PP, does show again an influence of memory for position, which is used here to refer to all features of the training situation, apart from target color, on which choice may be based (cf. Huber, Couvillon, \& Bitterman, 1994). Although the tendency to perseverate in terms of position is not strong enough here either to be itself detected or even to mask the tendency to perseverate in terms of color under conditions in which the two tendencies are frequently in conflict, it does serve to increase the probability of perseveration when the two tendencies consistently coincide.

That the marked color-position perseveration manifested in the confounded training is not to be understood as a conventional associative effect can be appreciated from the results for Group CfAi. The animals of that group were confronted on all trials by the same two alternatives (e.g., a blue target on the left and a yellow target on the right), which were equally often rewarded, and it is unlikely, from what we know of associative learning in honeybees, that single small rewards should continue to produce such marked shifts in preference (Couvillon \& Bitterman, 1991). Nor does it make sense in conventional associative terms that there should be perseveration on Trial 2 of a visit following small reward on Trial 1 but not on Trial 1 of a visit following much larger reward on Trial 2 of the preceding visit. It seems more reasonable to assume a short-term memory of the most recently rewarded alternative that generates a correspondingly transient preference for that alternative. Given the many similarities in the learning of honeybees and vertebrates (Bitterman, 1988, 1996), it is interesting to note that a like phenomenon (labeled positive recency) has been reported in recent work on color discrimination in pigeons (Bailey \& Mazur, 1990).

Working with honeybees in various six-alternative analogues of the rodent radial maze - the alternatives distinguished by landmarks differing both in color and shape - Brown and his colleagues (M. F. Brown \& Demas, 1994; M. F. Brown, Moore, C. H. Brown, \& Langheld, 1997) also have reported control of performance by shortterm or working memory, which appears in their data as a small tendency, present from the outset of training, to avoid recently rewarded alternatives. They attribute the meagerness of the effect to the poor discriminability of the targets they used, as shown by two experiments in which there was no decline with continued training in the number of visits to two of six targets that never contained sucrose. Working with several simpler radial-maze analoguesfewer alternatives distinguished only by position- 
Burmeister, Couvillon, and Bitterman (1995) found no such tendency but only some marked response biases. Whatever the source of the discrepancy in the results for the more complicated settings, it is difficult to understand why a tendency to avoid recently visited alternatives, if there is one, should not be evident in a simple two-choice situation with highly discriminable alternatives, such as the ones we used both here and in our prior experiments (Isnec et al., 1997; Ohyama et al., 1995).

Perhaps the most dramatic feature of the perseveration displayed by the confounded groups of the present experiment is its resistance to modification by differential reward. The perseverative tendency was neither strengthened by reward in Group CfP nor at all weakened by reward for alternation in Group CfA, which continued throughout the training to make many more errors than could be expected by chance. The ineffectiveness of the alternation training might be explained in terms of the strength of the original tendency to return to the locus of recent reward, but that does not account for the failure of the perseveration training to enhance the tendency. In further efforts to demonstrate learned control of performance by shortterm memory in honeybees, it will be important to find a way to subvert the initial perseverative tendency - as, for example, in "symbolic" matching (Carter \& Eckerman, 1975), where the sample to be remembered is different from either of the alternatives about the correctness of which it informs the subject.

\section{REFERENCES}

Bailey, J. T., \& MAzUR, J. E. (1990). Choice behavior in transition: Development of preference for the higher probability alternative. Journal of the Experimental Analysis of Behavior, 53, 409-422.

Bitterman, M. E. (1988). Vertebrate-invertebrate comparisons. In H. J. Jerison \& I. Jerison (Eds.), Intelligence and evolutionary biology (pp. 251-276). Berlin: Springer-Verlag.
Bitterman, M. E. (1996). Comparative analysis of learning in honeybees. Animal Learning \& Behavior, 24, 123-141.

Brown, M. F., \& Demas, G. E. (1994). Evidence for spatial working memory in honeybees (Apis mellifera). Journal of Comparative Psychology, 108, 344-352.

Brown, M. F., Moore, J. A., Brown, C. H., \& Langheld, K. D. (1997). The existence and extent of spatial working memory ability in honeybees. Animal Learning \& Behavior, 25, 472-484.

Burmeister, S., Couvillon, P. A., \& Bitterman, M. E. (1995). Performance of honeybees in analogues of the rodent radial maze. Animal Learning \& Behavior, 23, 369-375.

Carter, D. E., \& ECKERMAN, D. A. (1975). Symbolic matching by pigeons: Rate of learning complex discriminations predicted from simple discriminations. Science, 187, 662-664.

Couvillon, P. A., \& Bitterman, M. E. (1991). How honeybees make choices. In J. L. Goodman \& R. C. Fischer (Eds.), The behaviour and physiology of bees (pp. 116-130). Wallingford, U.K.: CAB International.

DENNIS, W. (1939). Spontaneous alternation in rats as an indicator of the persistence of stimulus effects. Journal of Comparative Psychology, 28, 305-312.

GiuRFA, M., \& NúÑEZ, J. A. (1992). Honeybees mark with scent and reject recently visited flowers. Oecologia, 89, 113-117.

Huber, B., Couvillon, P. A., \& Bitterman, M. E. (1994). Place and position learning in honeybees (Apis mellifera). Journal of Comparative Psychology, 108, 213-219.

Isnec, M. R., Couvillon, P. A., \& Bitterman, M. E. (1997). Shortterm spatial memory in honeybees. Animal Learning \& Behavior, 25 , 165-170.

NúÑEZ, J. A. (1967). Sammelbienen markieren versiegte Futterquellen durch Duft. Naturwissenschaften, 54, 322-323.

Ohyama, T., Couvillon, P. A., \& Bitterman, M. E. (1995). Perseveration in the color choices of honeybees. Journal of Insect Behavior, 8, 409-415.

Waddington, K. D., \& Gottlieb, N. (1990). Actual vs. perceived probability: A study of floral choice in honeybees. Journal of Insect Behavior, 3, 429-441.

(Manuscript received March 2, 1998; revision accepted for publication May 19, 1998.) 